\title{
Exercise-induced anaphylaxis: a case report and review of the literature
}

\author{
Saliha Esenboğa, Bülent Enis Şekerel \\ Division of Allergy and Asthma, Department of Pediatrics, Hacettepe University Faculty of Medicine, Ankara, Turkey. \\ E-mail: b sekerel@yahoo.com \\ Received: 20th March 2018, Revised: 29th June 2018, 24th July 2018, Accepted: 11th August 2018
}

SUMMARY: Esenboğa S, Şekerel BE. Exercise-induced anaphylaxis: a case report and review of the literature. Turk J Pediatr 2019; 61: 440-443.

Exercise-induced anaphylaxis (EIAn) is a rare, unpredictable, and potentially fatal cause of anaphylaxis which occurs with physical exhaustion. To the best of our knowledge, here we report the first case of EIAn from Turkey with no prior history of allergy. A detailed patient history is the most crucial point in the diagnosis of EIAn. The triggering and co-factors should be questioned in detail to determine treatment recommendations.

Key words: anaphylaxis, exercise.

Exercise-induced anaphylaxis (EIAn) is a rare, unpredictable, and potentially fatal cause of anaphylaxis which occurs with physical exhaustion. Maulitz et al. ${ }^{1}$ described it three decades ago, and the current information on the disease is mostly based on case reports.

There is an increase in the incidence rates especially in developed countries which may be attributed to the growing popularity of physical exercise and sports, as well as the cultural norms of being fit. ${ }^{2,3}$ Fatigue during or after varying degrees of exercise may trigger warmness, itching and erythema followed by urticaria(10-15 $\mathrm{mm}$ wide plaques) and angioedema. Continuing the exercise may cause gastrointestinal symptoms, laryngeal edema, hypotension, and collapse. ${ }^{4}$ After an attack, headache may take hours. The frequency of attacks is uncertain and varies from person to person. ${ }^{2}$

To the best of our knowledge, here we report the first case of EIAn from Turkey with no prior history of allergy.

\section{Case Report}

A 15-year-old girl was referred to the Hacettepe University Pediatric Allergy and Asthma Unit after a severe episode of anaphylaxis. She also had a history of recurrent episodes of flushing, generalized urticaria,and itching over the last 6 months'. In her medical history, it was reported that 7 years ago she had suffered from a skin rash and itching that started within 30 minutes after swimming(Fig. 1a) and spontaneously resolved in 15 minutes. In the following period, similar symptoms were repeated at irregular intervals, but both the severity and the duration of the reactions gradually increased over time. About one month prior to her referral she had to be taken to the emergency room due to nausea, dizziness, and skin rash which developed 2 to 3 minutes after swimming in the cold sea. At the emergency room, widespread urticaria, facial angioedema, and hypotension had been documented,and treated with repetitive adrenaline injections and intravenous fluid replacements.

The physical examination did not reveal any abnormality. The routine laboratory tests were normal. Detailed medical history excluded co-factors for EIAn or food dependent EIAn(FDEIAn); she did not consume alcohol or take any illicit drugs, there was no clue for concomitant infection including myocarditis. The patient did not have any chronic disease or use any medications. 


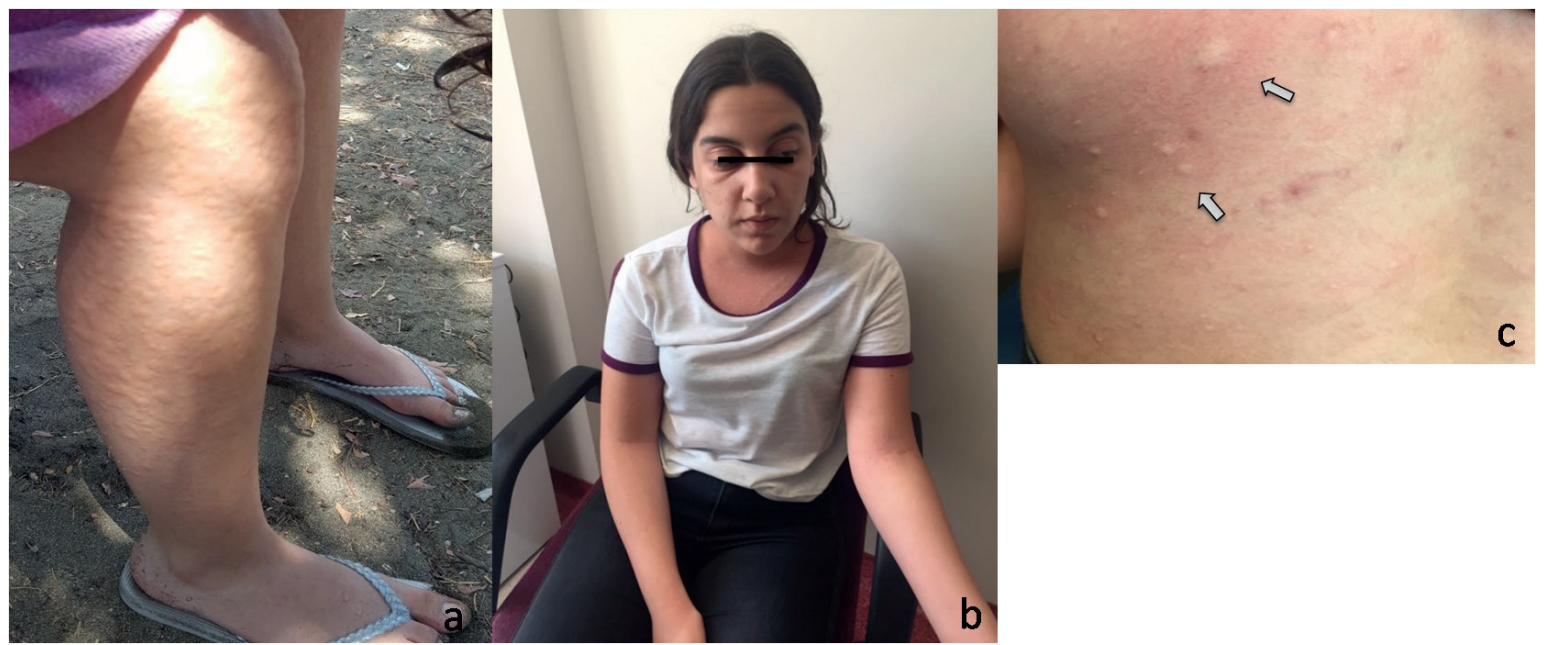

Fig. 1. a. Image of urticaria in the legs emerging after swimming b. Angioedema in the eyes during exercise provocation test $\mathbf{c}$. Urticarial plaques scattered around the body during exercise provocation test

There was no family history regarding allergic disease or atopy. Skin prick tests performed with food (wheat, gluten, rye, oat, barley, egg, milk, soy, peanut, hazelnut, sesame, etc.) and inhalant allergens (house dust mites, cat, dog, mold,etc.) were negative. Total IgE was 2,86 UI/mL. Specific IgE antibodies to wheat, gliadin and $\omega-5$ gliadin, cow's milk, egg yolk and white were all negative. 5 and 10 minutes' ice cube tests were negative. Baseline serum tryptase was $5,30 \mu \mathrm{g} / \mathrm{L}(<11.4 \mu \mathrm{g} / \mathrm{L})$. A fasting exercise challenge test on treadmill was performed in the morning. Within 2 minutes of the start of exercise provocation test, she experienced warmth and flushing and pruritus, then generalized urticaria, angioedema in the eyelids (Fig. 1b, c), sensation of fainting and hypotension $(60 / 30 \mathrm{mmHg})$. Gastrointestinal and respiratory symptoms did not occur. She was treated with multiple intramuscular adrenaline injections, fluid replacements parenteral methylprednisolone (60 mg), antihistamine (hyroxyzine $25 \mathrm{mg}$ ), and observed overnight on the ward. The symptoms disappeared within 2 hours. The tryptase level during anaphylaxis was 17.4 $\mu \mathrm{g} / \mathrm{L}, 1$ hour after anaphylaxis was 19,4 $\mu \mathrm{g} / \mathrm{L}$.

The patient was assessed with electrocardiography, echocardiography a 24hour holter monitorization to exclude a cardiac pathology. No cardiac problem was detected.

She was diagnosed with EIAn. The patient was asked to avoid heavy and long exercises and informed about triggering factors. An anaphylactic emergency action plan was prepared and epinephrine autoinjector was prescribed and its training was given. She was put on daily ketotifen treatment because she was overly afraid of another anaphylaxis event. During the next 2 months, the patient only underwent a brief urticarial episode at a time when she was not exercising. Two months later, ketotifen was stopped due to parents' concerns about weight gain and reduced school performance. After cessation of medication, she started to have complaints like warmth and tension sensation on her face, itching and utricaria while walking fast and climbing the stairs at school. She stated that she stopped the physical activity and would rest for a while when she felt warmth and itching.

Informed consent was obtained from the parents to use the photos of the patient.

\section{Discussion}

The patient presented above was diagnosed with EIAn. In the first minute of the exercise provocation test, classical symptoms of anaphylaxis such as urticaria, angioedema, and hypotension developed which required multiple adrenaline injections and intravenous hydration. Furthermore, increased levels of serum tryptase confirmed the diagnosis. Cold- and food dependent exercise-induced anaphlaxis were excluded with the patients's consistent history and positive exercise test. 
Anaphylaxis is an unpredictable, sudden, systemic hypersensitivity reaction affecting various organs and systems and has a potential risk forfatality. ${ }^{5}$ It is most often triggered by foods, medications, or venom, whereas physical factors such as exercise may also be a rare triggeringfactor. ${ }^{6,7}$ EIAn may occur as a resultof different forms of exercising(jogging, tennis, dancing, aerobics), whereas sometimes exercises like walking that cause less fatigue may also cause EIAn. ${ }^{4}$ Anaphylaxis in the presented patient appeared after swimming, climbing the stairs and walking fast.

The prevalence of the EIAn is unknown as the information on the subject is based on case reports. As it is the case of the presented patient, adolescents and young adults constitute the majority of reported cases. ${ }^{4}$ While the frequency of EIAn is similar in both sexes, FDEIAn is slightly more prevalent in men. ${ }^{8}$ It is known that patients' symptoms are better controlled over time due to better control of triggering factors.

There is great variability of symptoms in EIAn, hence it is unpredictable and difficult to diagnose. ${ }^{6}$ In a study conducted by Shaddick et $\mathrm{al}^{4}, 279$ patients were diagnosed with generalized pruritus and urticaria, flushing, and angioedema during the attack. Apart from these, headache, gastrointestinal colic, nausea,upper airway obstruction and dysphagia may occur as a sign of vascular compromise. Cessation of the exercise urgently helps to relieve the symptoms. However, at the first attack, the patient may not terminate the physical activity initially since the symptoms are experiencedfor the first time.

Although a clear pathophysiological mechanism that may explain EIAn has not yet been proven, there are several hypotheses such as increased gastrointestinal permeability, increased tissue transglutaminase activity in the intestinal mucosa, redistribution of blood during exercise and mast cell heterogeneity, increased plasma osmolality with exercise and triggering of basophil histamine release, triggering of acidosis and mast cell degranulation. ${ }^{9}$

The most critical point for EIAn is a detailed patient's history. The warmth, erythema, itching, and hives triggered by exercise highly support the exercise-induced urticaria or anaphylaxis. Progression of the symptoms to dysphagia, dyspnea, wheezing, dizziness, and fainting is compatible with EIAn. Symptoms disappear 30 minutes to 4 hours after finishing the exercise. ${ }^{4}$ There should be no other diagnosis that could explain this clinical picture. Physical examination should be done carefully for urticaria pigmentosamastocytosis. If the exercise provocation is positive as in the presented patient, the diagnosis is clear ${ }^{10}$,but if it is negative, the diagnosis is not excluded. For this reason, before the exercise test, the benefit of diagnosis should be compared with the risk of triggering anaphylactic reactions and written informed consent should be signed by the patient. During the test, the patient must be under close observation. Basal serum tryptase, skin prick test, specific IgE for wheat, gluten and $\omega-5$ gliadin-specific IgE should be assessed absolutely in terms of differential diagnosis and facilitating factors.

Differential diagnosis includes cholinergic urticaria, food-dependent exercise-induced urticaria, cold-induced urticaria/anaphylaxis, mastocytosis, cardiovascular events etc. In cholinergic urticaria, urticarial plaques can be triggered by heat and sweating and are seen as pinpoint ${ }^{11}$ whereas the presented patient had larger urticarial plaques. Since there were no typical history suggesting FDEIAn or specific IgE or skin prick test positivity with a culprit food (most commonly wheat), FDEIAn was excluded. The normal basal serum tryptase enabled us to eliminate mast cell diseases. The ice cube test to distinguish it from cold urticaria was negative. Cardiovascular events were excluded,given that the cardiac enzymes during reaction, electrocardiogram, echocardiography and 24-hour Holter monitorization were all normal.

An anaphylaxis emergency action plan was prepared for the patient, the epinephrine autoinjector was prescribed and she was trained for its use. She was treated with ketotifen, a mast cell stabilizer and antihistamine. There are no randomized controlled trials for treatment options. The level of evidence for treatment is low, and the recommended treatments are usually based on 
clinical experience. Patient-specific treatment approach should be preferred. Prevention of triggers, cofactors such as diminishing the exercise intensity and prevention of preexercise eating is the first step to be taken. $\mathrm{H} 1$ antihistamines are recommended as prophylactic pharmacotherapy 2 hours before exercise. There are successful case reports regarding omalizumab in the treatment of EIAn. ${ }^{12}$ One report presented montelukast in combination with Cetirizine. ${ }^{10}$

EIAn is a rare disorder and should be carefully identified to prevent life-threatening anaphylactic reactions. A detailed patient history is the most crucial point in the diagnosis. The triggering and co-factors should be questioned in detail to determine treatment recommendations.

\section{REFERENCES}

1. Maulitz RM, Pratt DS, Schocket AL. Exercise-induced anaphylactic reaction to shellfish. J Allergy Clin Immunol 1979; 63:433-434.

2. Wylon K, Hompes S, Worm M. Exercise-induced anaphylaxis. Hautarzt 2013; 64: 97-101.

3. Bennett JR. Anaphylaxis attributed to exercise: Considerations for sports medicine specialists. Phys Sportsmed 2015; 43:1-12.

4. Shadick NA, Liang MH, Partridge AJ, et al. The natural history of exercise-induced anaphylaxis: Survey results from a 10-year follow-up study. J Allergy Clin Immunol 1999; 104: 123-127.
5. Johansson SG, Bieber T, Dahl R, et al. Revised nomenclature for allergy for global use: Report of the Nomenclature Review Committee of the World Allergy Organization, October 2003. J Allergy Clin Immunol 2004; 113: 832-836.

6. Sampson HA, Munoz-Furlong A, Campbell RL, et al. Second symposium on the definition and management of anaphylaxis: Summary report--second National Institute of Allergy and Infectious Disease/Food Allergy and Anaphylaxis Network symposium. Ann Emerg Med 2006; 47: 373-380.

7. Soyer OU, Sekerel BE. Food dependent exercise induced anaphylaxis or exercise induced anaphylaxis? Allergol Immunopathol (Madr) 2008; 36: 242-243.

8. Beaudouin E, Renaudin JM, Morisset M, Codreanu F, Kanny G, Moneret-Vautrin DA. Food-dependent exercise-induced anaphylaxis--update and current data. Eur Ann Allergy Clin Immunol 2006; 38: 45-51.

9. Ansley L, Bonini $\mathrm{M}$, Delgado $\mathrm{L}$, et al. Pathophysiological mechanisms of exercise-induced anaphylaxis: An EAACI position statement. Allergy 2015; 70: 1212-1221.

10. Gajbhiye S, Agrawal RP, Atal S, Tiwari V, Phadnis P. Exercise-induced anaphylaxis and antileukotriene montelukast. J Pharmacol Pharmacother 2015; 6: 163-165.

11. Montgomery SL. Cholinergic urticaria and exerciseinduced anaphylaxis. Curr Sports Med Rep 2015; 14: 61-63.

12. Bray SM, Fajt ML, Petrov AA. Successful treatment of exercise-induced anaphylaxis with omalizumab. Ann Allergy Asthma Immunol 2012; 109: 281-282. 